\title{
A CORRECTION TO APPROXIMATION OF GENERALIZED HOMOMORPHISMS IN QUASI-BANACH ALGEBRAS
}

\author{
ISMAIL NIKOUFAR
}

Received 23 June, 2016

\begin{abstract}
Eshaghi et. al [Approximation of generalized homomorphisms in quasi-Banach algebras, An. St. Univ. Ovidius Constanta, 17(2), (2009), 203-214] defined the notion of generalized homomorphisms in quasi-Banach algebras. They investigated generalized homomorphisms from quasi-Banach algebras to $p$-Banach algebras and proved the generalized Hyers-Ulam-Rassias stability. In this paper, we show that their results only hold for Banach algebras and then we correct and prove the results for $p$-Banach algebras.
\end{abstract}

2010 Mathematics Subject Classification: 46B03; 47Jxx; 47B48; 39B52

Keywords: Hyers-Ulam-Rassias stability, quasi-Banach algebra, $p$-Banach algebra, generalized homomorphism

\section{INTRODUCTION AND PRELIMINARIES}

We know that a $\mathbb{C}$-linear mapping $f: A \rightarrow B$ is called a homomorphism if $f(x y)=$ $f(x) f(y)$ for all $x, y \in A$ so that every homomorphism is a generalized homomorphism, but the converse is false, in general.

Eshaghi et. al [1] investigated generalized homomorphisms from quasi-Banach algebras to $p$-Banach algebras and proved the generalized Hyers-Ulam-Rassias stability. In this paper, we verify that the results presented in [1] hold for Banach algebras. Then, we correct their results and prove the results for $p$-Banach algebras. We remark that the presenting results in this paper hold for $p$-Banach algebras, where $0<p \leq 1$, in general. The stability problems of several functional equations have been extensively investigated by a number of authors in $p$-Banach algebras and there are many interesting results concerning this problem (see [2,3,5] and references therein).

Let $X$ be a real linear space. A quasi-norm is a real-valued function on $X$ satisfying the following conditions:

(i) $\|x\| \geq 0$ for all $x \in X$ and $\|x\|=0$ if and only if $x=0$,

(ii) $\|\lambda x\|=|\lambda|\|x\|$ for all $\lambda \in \mathbb{R}$ and all $x \in X$,

(iii) there is a constant $K \geq 1$ such that $\|x+y\| \leq K(\|x\|+\|y\|)$ for all $x, y \in X$. 
The pair $(X,\|\cdot\|)$ is called a quasi-normed space if $\|\cdot\|$ is a quasi-norm on $X$. The smallest possible $K$ is called the modulus of concavity of $\|\cdot\|$. A quasi-Banach space is a complete quasi-normed space. Indeed, by a quasi-Banach space we mean a quasi-normed space in which every $\|\cdot\|-$ Cauchy sequence in $X$ converges. This class includes Banach spaces and the most significant class of quasi-Banach spaces which are not Banach spaces. A quasi-norm $\|\cdot\|$ is called a $p$-norm $(0<p \leq 1)$ if

$$
\|x+y\|^{p} \leq\|x\|^{p}+\|y\|^{p}
$$

for all $x, y \in X$. In this case, a quasi-Banach space is called a $p$-Banach space.

Let $(A,\|\cdot\|)$ be a quasi-normed space. The quasi-normed space $(A,\|\cdot\|)$ is called a quasi-normed algebra if $A$ is an algebra and there is a constant $K>0$ such that $\|x y\| \leq K\|x\|\|\mid y\|$ for all $x, y \in A$. A quasi-Banach algebra is a complete quasinormed algebra. If the quasi-norm $\|\cdot\|$ is a $p$-norm, then the quasi-Banach algebra is called a $p$-Banach algebra. Eshaghi et. al [1] defined the notion of generalized homomorphisms in quasi-Banach algebra as follows:

Definition 1. Let $A$ be a quasi-Banach algebra with quasi-norm $\|\cdot\|_{A}$ and let $B$ be a $p$-Banach algebra with $p$-norm $\|\cdot\|_{B}$. A $\mathbb{C}$-linear mapping $f: A \rightarrow B$ is called a generalized homomorphism if there exists a homomorphism $h: A \rightarrow B$ such that $f(x y)=f(x) h(y)$ for all $x, y \in A$.

Then, they investigated generalized homomorphisms from quasi-Banach algebras to $p$-Banach algebras associated with the following functional equation

$$
r f\left(\frac{x+y}{r}\right)=f(x)+f(y)
$$

and proved the generalized Hyers-Ulam-Rassias stability and superstability of generalized homomorphisms in quasi-Banach algebras. In this paper, we prove that their results only hold for Banach algebras and then we correct their results and confirm the results for $p$-Banach algebras.

\section{MAIN PROBLEMS}

Following [1] throughout this paper, assume that $A$ is a quasi-Banach algebra with quasi-norm $\|\cdot\|_{A}$ and that $B$ is a $p$-Banach algebra with $p$-norm $\|\cdot\|_{B}$. In addition, we assume $r$ to be a constant positive integer.

We will use the following lemma in this section.

Lemma 1 ([4]). Let $X$ and $Y$ be linear spaces and let $f: X \rightarrow Y$ be an additive mapping such that $f(\mu x)=\mu f(x)$ for all $x \in X$ and $\mu \in \mathbb{T}^{1}:=\{z \in \mathbb{C}:|z|=1\}$. Then the mapping $f$ is $\mathbb{C}$-linear.

The following two theorems proved in [1, Theorems 2.2 and 2.5]. In these theorems, the authors want to prove the generalized Hyers-Ulam-Rassias stability of generalized homomorphisms from quasi-Banach algebras to $p$-Banach algebras. 
Theorem 1. Suppose $f: A \rightarrow B$ is a mapping with $f(0)=0$ for which there exist a mapping $g: A \rightarrow B$ with $g(0)=0, g(1)=1$ and a function $\varphi: A^{4} \rightarrow \mathbb{R}^{+}$such that

$$
\begin{gathered}
\left\|r f\left(\frac{\mu a+\mu b+c d}{r}\right)-\mu f(a)-\mu f(b)-f(c) g(d)\right\|_{B} \leq \varphi(a, b, c, d), \\
\|g(\mu a b+\mu c d)-\mu g(a) g(b)-\mu g(c) g(d)\|_{B} \leq \varphi(a, b, c, d),
\end{gathered}
$$

and

$$
\tilde{\varphi}(a, b, c, d):=\sum_{i=0}^{\infty} \frac{\varphi\left(2^{i} a, 2^{i} b, 2^{i} c, 2^{i} d\right)}{2^{i}}<\infty
$$

for all $a, b, c, d \in A$ and all $\mu \in \mathbb{T}^{1}$. Then, there exists a unique generalized homomorphism $h: A \rightarrow B$ such that

$$
\|f(a)-h(a)\|_{B} \leq \frac{1}{2} \tilde{\varphi}(a, a, 0,0)
$$

for all $a \in A$.

Theorem 2. Suppose $f: A \rightarrow B$ is a mapping with $f(0)=0$ for which there exist a mapping $g: A \rightarrow B$ with $g(0)=0, g(1)=1$ and a function $\varphi: A^{4} \rightarrow \mathbb{R}^{+}$satisfying the inequalities (2.1), (2.2) and

$$
\tilde{\varphi}(a, b, c, d):=\sum_{i=1}^{\infty} 2^{i} \varphi\left(\frac{a}{2^{i}}, \frac{b}{2^{i}}, \frac{c}{2^{i}}, \frac{d}{2^{i}}\right)<\infty
$$

for all $a, b, c, d \in A$ and all $\mu \in \mathbb{T}^{1}$. Then, there exists a unique generalized homomorphism $h: A \rightarrow B$ such that

$$
\|f(a)-h(a)\|_{B} \leq \frac{1}{2} \tilde{\varphi}(a, a, 0,0)
$$

for all $a \in A$.

In the following theorems we correct the results and prove the generalized HyersUlam-Rassias stability of generalized homomorphisms from quasi-Banach algebras to $p$-Banach algebras.

Theorem 3. Suppose $f: A \rightarrow B$ is a mapping with $f(0)=0$ for which there exist a mapping $g: A \rightarrow B$ with $g(0)=0, g(1)=1$ and a function $\varphi: A^{4} \rightarrow \mathbb{R}^{+}$such that

$$
\begin{gathered}
\left\|r f\left(\frac{\mu a+\mu b+c d}{r}\right)-\mu f(a)-\mu f(b)-f(c) g(d)\right\|_{B} \leq \varphi(a, b, c, d), \\
\|g(\mu a b+\mu c d)-\mu g(a) g(b)-\mu g(c) g(d)\|_{B} \leq \varphi(a, b, c, d),
\end{gathered}
$$

and

$$
\tilde{\varphi}(a, b, c, d):=\left(\sum_{i=0}^{\infty} \frac{\varphi\left(2^{i} a, 2^{i} b, 2^{i} c, 2^{i} d\right)^{p}}{2^{i p}}\right)^{\frac{1}{p}}<\infty
$$


for all $a, b, c, d \in A$ and all $\mu \in \mathbb{T}^{1}$. Then, there exists a unique generalized homomorphism $h: A \rightarrow B$ such that

$$
\|f(a)-h(a)\|_{B} \leq \frac{1}{2} \tilde{\varphi}(a, a, 0,0)
$$

for all $a \in A$.

Proof. Setting $a=b, c=d=0$ and $r=\mu=1$ in (2.3) and dividing both sides of the resulting inequality by 2 , we obtain

$$
\left\|\frac{f(2 a)}{2}-f(a)\right\|_{B} \leq \frac{\varphi(a, a, 0,0)}{2}
$$

for all $a \in A$. Then, we have

$$
\left\|\frac{f(2 a)}{2}-f(a)\right\|_{B}^{p} \leq \frac{\varphi(a, a, 0,0)^{p}}{2^{p}}
$$

for all $a \in A$. Replacing $a$ in (2.7) by $2 a$ and dividing both sides of the resulting inequality by 2 , we get

$$
\left\|\frac{f\left(2^{2} a\right)}{2^{2}}-\frac{f(2 a)}{2}\right\|_{B} \leq \frac{\varphi(2 a, 2 a, 0,0)}{2^{2}}
$$

for all $a \in A$. Then, we have

$$
\left\|\frac{f\left(2^{2} a\right)}{2^{2}}-\frac{f(2 a)}{2}\right\|_{B}^{p} \leq \frac{\varphi(2 a, 2 a, 0,0)^{p}}{2^{2 p}}
$$

for all $a \in A$. Applying (2.8), (2.10), and (1.1) we detect that

$$
\left\|\frac{f\left(2^{2} a\right)}{2^{2}}-f(a)\right\|_{B}^{p} \leq \frac{\varphi(a, a, 0,0)^{p}}{2^{p}}+\frac{\varphi(2 a, 2 a, 0,0)^{p}}{2^{2 p}}
$$

for all $a \in A$. By induction on $n$ we conclude that

$$
\left\|\frac{f\left(2^{n} a\right)}{2^{n}}-f(a)\right\|_{B}^{p} \leq \frac{1}{2^{p}} \sum_{i=0}^{n-1} \frac{\varphi\left(2^{i} a, 2^{i} a, 0,0\right)^{p}}{2^{i p}}
$$

for all $a \in A$ and all non-negative integers $n$. Consequently,

$$
\left\|\frac{f\left(2^{n+m} a\right)}{2^{n+m}}-\frac{f\left(2^{m} a\right)}{2^{m}}\right\|_{B} \leq \frac{1}{2}\left(\sum_{i=m}^{n+m-1} \frac{\varphi\left(2^{i} a, 2^{i} a, 0,0\right)^{p}}{2^{i p}}\right)^{\frac{1}{p}}
$$

for all non-negative integers $n$ and $m$ with $n \geq m$ and all $a \in A$. It follows from (2.5) and (2.13) that the sequence $\left\{\frac{f\left(2^{n} a\right)}{2^{n}}\right\}$ is Cauchy in $B$ for all $a \in A$. Since $B$ is a $p$-Banach algebras, this sequence is convergent in $B$ for all $a \in A$. Define the mapping

$$
h(a):=\lim _{n \rightarrow \infty} \frac{f\left(2^{n} a\right)}{2^{n}} .
$$


Setting $c=d=0, r=1$ and replacing $a, b$ by $2^{n} a, 2^{n} b$, respectively, in (2.3) and dividing both sides of (2.3) by $2^{n}$ and taking the limit as $n \rightarrow \infty$ we deduce

$$
h(\mu a+\mu b)=\mu h(a)+\mu h(b)
$$

for all $a, b \in A$ and $\mu \in \mathbb{T}^{1}$. So the mapping $h$ is $\mathbb{C}$-linear by Lemma 1 . Note that inequality (2.6) follows from (2.12) and (2.14). To show that $h$ is unique, let $k$ be another $\mathbb{C}$-linear mapping satisfying (2.6). From (2.6) we conclude that

$$
\begin{aligned}
\|h(a)-k(a)\|_{B}^{p} & =\frac{1}{2^{n p}}\left\|h\left(2^{n} a\right)-k\left(2^{n} a\right)\right\|_{B}^{p} \\
& \leq \frac{1}{2^{n p}}\left(\left\|h\left(2^{n} a\right)-f\left(2^{n} a\right)\right\|_{B}^{p}+\left\|f\left(2^{n} a\right)-k\left(2^{n} a\right)\right\|_{B}^{p}\right. \\
& \leq \frac{1}{2^{n p}} \frac{2}{2^{p}} \tilde{\varphi}\left(2^{n} a, 2^{n} a, 0,0\right)^{p} \\
& =\frac{2}{2^{p}} \sum_{i=n}^{\infty} \frac{\varphi\left(2^{i} a, 2^{i} a, 0,0\right)^{p}}{2^{i p}}
\end{aligned}
$$

for all $a \in A$. The right hand side tends to zero as $n \rightarrow \infty$. The rest of the proof is similar to that of [1, Theorem 2.2] and we omit it.

Theorem 4. Suppose $f: A \rightarrow B$ is a mapping with $f(0)=0$ for which there exist a mapping $g: A \rightarrow B$ with $g(0)=0, g(1)=1$ and a function $\varphi: A^{4} \rightarrow \mathbb{R}^{+}$satisfying the inequality (2.3) and (2.4) and

$$
\tilde{\varphi}(a, b, c, d):=\left(\sum_{i=1}^{\infty} 2^{i p} \varphi\left(\frac{a}{2^{i}}, \frac{b}{2^{i}}, \frac{c}{2^{i}}, \frac{d}{2^{i}}\right)^{p}\right)^{\frac{1}{p}}<\infty
$$

for all $a, b, c, d \in A$ and all $\mu \in \mathbb{T}^{1}$. Then, there exists a unique generalized homomorphism $h: A \rightarrow B$ such that

$$
\|f(a)-h(a)\|_{B} \leq \frac{1}{2} \tilde{\varphi}(a, a, 0,0)
$$

for all $a \in A$.

Proof. Setting $a=b, c=d=0$ and $r=\mu=1$ in (2.3), we obtain

$$
\|f(2 a)-2 f(a)\|_{B} \leq \varphi(a, a, 0,0)
$$

for all $a \in A$. Replacing $a$ in (2.18) by $\frac{a}{2}$, we get

$$
\left\|f(a)-2 f\left(\frac{a}{2}\right)\right\|_{B} \leq \varphi\left(\frac{a}{2}, \frac{a}{2}, 0,0\right)
$$

for all $a \in A$. Replacing $a$ in (2.19) by $\frac{a}{2}$ and multiplying both sides of the resulting inequality by 2 , we detect

$$
\left\|2 f\left(\frac{a}{2}\right)-2^{2} f\left(\frac{a}{2^{2}}\right)\right\|_{B} \leq 2 \varphi\left(\frac{a}{2^{2}}, \frac{a}{2^{2}}, 0,0\right)
$$


for all $a \in A$. Using (2.19), (2.20), and (1.1) we conclude that

$$
\left\|f(a)-2^{2} f\left(\frac{a}{2^{2}}\right)\right\|_{B}^{p} \leq \varphi\left(\frac{a}{2}, \frac{a}{2}, 0,0\right)^{p}+2^{p} \varphi\left(\frac{a}{2^{2}}, \frac{a}{2^{2}}, 0,0\right)
$$

for all $a \in A$. By induction on $n$ we deduce that

$$
\left\|f(a)-2^{n} f\left(\frac{a}{2^{n}}\right)\right\|_{B}^{p} \leq \frac{1}{2^{p}} \sum_{i=1}^{n} 2^{i p} \varphi\left(\frac{a}{2^{i}}, \frac{a}{2^{i}}, 0,0\right)^{p}
$$

for all $a \in A$ and all non-negative integers $n$. Hence,

$$
\left\|2^{m} f\left(\frac{a}{2^{m}}\right)-2^{n+m} f\left(\frac{a}{2^{n+m}}\right)\right\|_{B}^{p} \leq \frac{1}{2^{p}} \sum_{i=m+1}^{m+n} 2^{i p} \varphi\left(\frac{a}{2^{i+m}}, \frac{a}{2^{i+m}}, 0,0\right)^{p}
$$

for all non-negative integers $n$ and $m$ with $n \geq m$ and all $a \in A$. It follows from (2.16) and (2.23) that the sequence $\left\{2^{n} f\left(\frac{a}{2^{n}}\right)\right\}$ is Cauchy in $B$ for all $a \in A$ so that this sequence is convergent in $B$. Define the mapping

$$
h(a):=\lim _{n \rightarrow \infty} 2^{n} f\left(\frac{a}{2^{n}}\right) .
$$

The rest of the proof is similar to that of Theorem 3 and we omit it.

Corollary 1. Suppose $f: A \rightarrow B$ is a mapping with $f(0)=0$ for which there exist constants $\epsilon>0, \alpha \neq 1$ and a mapping $g: A \rightarrow B$ with $g(0)=0, g(1)=1$ such that

$$
\begin{gathered}
\left\|r f\left(\frac{\mu a+\mu b+c d}{r}\right)-\mu f(a)-\mu f(b)-f(c) g(d)\right\|_{B} \\
\leq \epsilon\left(\|a\|_{A}^{\alpha}+\|b\|_{A}^{\alpha}+\|c\|_{A}^{\alpha}+\|d\|_{A}^{\alpha}\right),
\end{gathered}
$$

$\|g(\mu a b+\mu c d)-\mu g(a) g(b)-\mu g(c) g(d)\|_{B} \leq \epsilon\left(\|a\|_{A}^{\alpha}+\|b\|_{A}^{\alpha}+\|c\|_{A}^{\alpha}+\|d\|_{A}^{\alpha}\right)$ for all $a, b, c, d \in A$ and all $\mu \in \mathbb{T}^{1}$. Then, there exists a unique generalized homomorphism $h: A \rightarrow B$ such that

$$
\|f(a)-h(a)\|_{B} \leq \frac{\epsilon}{\left|1-2^{p(\alpha-1)}\right|^{\frac{1}{p}}}\|a\|_{A}^{\alpha} .
$$

for all $a \in A$.

Proof. Define $\varphi(a, b, c, d):=\epsilon\left(\|a\|_{A}^{\alpha}+\|b\|_{A}^{\alpha}+\|c\|_{A}^{\alpha}+\|d\|_{A}^{\alpha}\right)$. If $0 \leq \alpha<1$, then Theorem 3 entails that

$$
\tilde{\varphi}(a, a, 0,0)=\frac{2 \epsilon}{\left(1-2^{p(\alpha-1)}\right)^{\frac{1}{p}}}\|a\|_{A}^{\alpha} .
$$

Consequently,

$$
\|f(a)-h(a)\|_{B} \leq \frac{\epsilon}{\left(1-2^{p(\alpha-1)}\right)^{\frac{1}{p}}}\|a\|_{A}^{\alpha} .
$$


If $\alpha>1$, then by applying Theorem 4 we find that

$$
\tilde{\varphi}(a, a, 0,0)=\frac{2 \epsilon}{\left(2^{p(\alpha-1)}-1\right)^{\frac{1}{p}}}\|a\|_{A}^{\alpha} .
$$

Hence,

$$
\|f(a)-h(a)\|_{B} \leq \frac{\epsilon}{\left(2^{p(\alpha-1)}-1\right)^{\frac{1}{p}}}\|a\|_{A}^{\alpha} .
$$

From inequalities (2.26) and (2.27) we conclude inequality (2.25).

\section{CONCLUSiOnS}

We conclude that

(i) in Theorems 3, 4, if we take $p=1$, then we obtain [1, Theorems 2.2, 2.5], respectively,

(ii) in Corollary 1, if we take $p=1$, then we deduce [1, Corollary 2.3],

(iii) in Corollary 1 , if we take $p=1, \epsilon=\frac{\delta}{2}$, and $\alpha=0$, then we recover [1, Corollary 2.4].

Indeed, the results presented in [1] hold for 1-Banach algebras. We know that 1Banach algebras exactly coincide with Banach algebras and so the results of [1] only hold for Banach algebras. Our presented results in this paper hold for $p$-Banach algebras where $0<p \leq 1$.

\section{ACKNOWLEDGMENTS}

This research was supported by a grant from Payame Noor University with the same title. The author would like to thank the referee for the careful reading of the paper.

\section{REFERENCES}

[1] M. Eshaghi Gordji and M. B. Savadkouhi, "Approximation of generalized homomorphisms in quasi-Banach algebras," An. St. Univ. Ovidius Constanta, vol. 17, no. 2, pp. 203-214, 2009.

[2] A. Najati, "Homomorphisms in quasi-Banach algebras associated with a Pexiderized CauchyJensen functional equation," Acta Math. Sinica, vol. 25, no. 9, pp. 1529-1542, 2009.

[3] A. Najati and C. Park, "Hyers-Ulam-Rassias stability of homomorphisms in quasi-Banach algebras associated to the Pexiderized Cauchy functional equation," J. Math. Anal. Appl., vol. 335, pp. 763-778, 2007.

[4] C. Park, "Homomorphisms between Poisson JC*-algebras," Bull. Braz. Math. Soc., vol. 36, pp. 79-97, 2005.

[5] C. Park, "Hyers-Ulam-Rassias stability of homomorphisms in quasi-Banach algebras," Bull. Sci. Math., vol. 132, pp. 87-96, 2008. 
Author's address

\section{Ismail Nikoufar}

Department of Mathematics, Payame Noor University, P.O. BOX 19395-3697 Tehran, Iran E-mail address: nikoufar@pnu.ac.ir 